Esta revista forma parte del acervo de la Biblioteca Jurídica Virtual del Instituto de Investigaciones Jurídicas de la UNAM www.juridicas.unam.mx

http://biblio.juridicas.unam.mx

\title{
Perspectivas y evaluación del modelo de comunicación político electoral en México
}

\author{
Aleida Elsi Calleja Gutiérrez* \\ Beatriz Solís Leere** \\ Aimée Vega Montiel*** \\ Rebeca Domínguez Cortina**** \\ Jorge Enrique Bravo Torres Coto***** \\ Agustín Ramírez Ramírez****** \\ José Agustín Pineda Ventura*******
}

\section{Sumario:}

I. Introducción

II. Principales hallazgos de las líneas de investigación

III. Análisis jurídico: la norma electoral y las resoluciones

de las autoridades en materia de libertad de expresión

IV. Fuentes de consulta

* Licenciada en Ciencias de la Comunicación por la Universidad Iberoamericana; presidenta de la AMEDI.

** Profesora-investigadora en la UAM-Xochimilco; coordinadora del Comité Relaciones Internacionales de la AMEDI.

*** Doctora en Periodismo y Comunicación de la Universidad Autónoma de Barcelona, maestra en Ciencias de la Comunicación de la UNAM; directora de la AMEDI.

$* * * *$ Maestra en Ciencias de la Comunicación por la UNAM.

$* * * * *$ Maestro en Comunicación por la UNAM; coordinador del Comité Editorial de la AMEDI. $x_{* * * * *}$ Licenciado en Derecho,por la UAM-Azcapotzalco; consejero consultivo de la AMEDI.

$* \star * \star * \star *$ Licenciado en Derecho por la UAM-Azcapotzalco; coordinador del Comité Jurídico de la AMEDI. 
Esta revista forma parte del acervo de la Biblioteca Jurídica Virtual del Instituto de Investigaciones Jurídicas de la UNAM www.juridicas.unam.mx

\section{Resumen:}

La reforma electoral de 2007-2008 estableció un cambio sustancial en las reglas del modelo de comunicación político electoral al prohibir la contratación de tiempos en radio y televisión. Como medida para garantizar la equidad en los procesos electorales del acceso a los medios electrónicos por parte de candidaturas y partidos políticos se estableció la utilización de los tiempos de Estado, de esta manera se trató de inhibir la injerencia del poder del dinero en la democracia. Esta prohibición canceló millonarias ganancias a los concesionarios comerciales de la radio y la televisión, que de manera constante han acusado a la ley y a las autoridades electorales de violar la libertad de expresión.

Con el objetivo de evaluar la pertinencia del actual modelo de comunicación política electoral para la democracia mexicana, así como determinar si la ley o las autoridades violan la libertad de expresión, la Asociación Mexicana de Derecho a la Información (AMEDI), realizó un análisis jurídico sobre las resoluciones de la autoridad en casos paradigmáticos relacionados con la libertad de expresión, así como el comportamiento de los medios y las formas de comunicación de partidos y candidatos a través de la radio y la televisión a través de un Observatorio de Medios.

Palabras clave: comunicación político electoral, libertad de expresión, derecho a la información, observación de medios, autoridades electorales, debates, tiempos de Estado. 


\section{Introducción}

La reforma constitucional de 2007-2008 en materia electoral, cambió las reglas del modelo de comunicación político electoral en México al determinar la prohibición de compra de espacios para la transmisión de campañas en radio y televisión, con el propósito de garantizar la equidad entre los actores políticos para el acceso a los medios electrónicos y evitar que el dinero fuera el elemento determinante para poder dar a conocer la oferta electoral, así como que instancias ajenas a los procesos comiciales tuvieran injerencias indebidas para incidir en las preferencias del voto.

La reforma también dio respuesta a la demanda de diversos sectores de la sociedad para que las campañas electorales fueran más cortas y se redujera el desmedido gasto de recursos públicos por parte de los partidos políticos, que en su mayor parte erogaban sus gastos en la contratación de spots en los medios electrónicos. De acuerdo con varias investigaciones, 7 de cada 10 pesos de financiamiento público para las campañas electorales se destinaban a la propaganda en medios electrónicos. De esta manera, el modelo de comunicación social cambió para evitar que ni el dinero ni el poder de los medios de comunicación se convirtiera en factores determinantes de las campañas electorales y sus resultados.

La relación entre dinero, medios y democracia en el modelo de comunicación política en nuestro país ha tenido una preeminencia importante para determinar la equidad en las contiendas, y ha tenido un impacto ineludible en los ámbitos del derecho a la información y la libertad de expresión.

La creciente influencia de la radio y la televisión en los procesos electorales, ha generado efectos contrarios a la democracia al propiciar la adopción, consciente o no, de patrones de propaganda política y electoral que imitan o reproducen los utilizados en el mercado para la promoción de mercancías y servicios que se pretende acepten los consumidores.

En México, este hecho se vio ampliamente reflejado durante las elecciones de 2006, donde se hizo evidente que la alta compra de espacios para la transmisión de spots por parte de los partidos políticos 
Esta revista forma parte del acervo de la Biblioteca Jurídica Virtual del Instituto de Investigaciones Jurídicas de la UNAM

benefició esencialmente a los medios electrónicos, especialmente a las dos grandes televisoras del país: Televisa y TV Azteca.

Bajo tales tendencias, la política y la competencia electoral van quedando sujetas no solamente a modelos de propaganda que les son ajenos, sino también al riesgo de la influencia de los dueños o concesionarios de estaciones de radio y canales de televisión (o de otros grupos con el poder económico necesario para reflejarlo en esos medios de comunicación) en sus respectivas audiencias, al resaltar la imagen de aquellos candidatos a un puesto de elección popular que sean afines a sus intereses o garanticen el fortalecimiento de sus negocios, lo cual deriva en un poder fáctico contrario al orden democrático constitucional.

Al ver cancelado un millonario negocio por la venta de espacios en las campañas electorales, los concesionarios privados de la radiodifusión, encabezados por las principales televisoras del país, han tenido una decidida oposición a las nuevas reglas, por lo que han implementado estrategias jurídicas y mediáticas para evitar su cumplimiento y para acusar que tanto la nueva ley electoral, como el Instituto Federal Electoral (IFE) violan la libertad de expresión. Con este argumento sustentan su principal demanda de cambiar la nueva ley, para lo que muchos suponemos que lo que está en el fondo es volver al modelo comercial de comunicación política electoral.

Si reconocemos que tanto la libertad de expresión como el derecho a la información son derechos pivote que permiten el ejercicio de otros derechos humanos y ciudadanos, al permitir las condiciones para un debate democrático entre las distintas fuerzas y sectores de la sociedad para sustentar el derecho a elegir libremente, resulta de primordial importancia el estudio de su ejercicio y condiciones en el marco del actual modelo de comunicación político electoral.

Frente a las constantes descalificaciones y críticas sobre las afectaciones que este nuevo modelo de comunicación política puede tener en la libertad de expresión, y por ende en el derecho a la información, la Asociación Mexicana de Derecho a la Información (AMEDI) realizó una investigación con el fin de determinar, con elementos consistentes de análisis, las afectaciones a tales derechos, el cual se dividió en los siguientes componentes y líneas de investigación: 


\section{Componente observatorio de medios}

A. Objeto y formas de la propaganda electoral

Consistente en monitorear y analizar el contenido y sentido de la propaganda político-electoral de los candidatos a la Presidencia transmitida por televisión y radio durante el proceso electoral de 2012 y verificar su contribución a un modelo de comunicación política democrático.

B. Ejercicio periodístico y campañas electorales en el marco de la libertad de expresión y derecho a la información

Consistente en monitorear y analizar los contenidos de los programas de opinión y análisis trasmitidos en televisión respecto a los candidatos a la Presidencia de la República como al marco jurídico electoral y a las autoridades respectivas.

C. Debates y libertad de expresión

Consistente en monitorear y evaluar el esquema con el que se desarrollaron los debates presidenciales en el marco de la reforma electoral, así como los programas de pre y pos debate transmitidos por televisión.

\section{Componente de análisis jurídico}
A. Los límites a la libertad de expresión en la interpretación de las autoridades electorales

Su propósito es analizar las resoluciones del TribunalElectoral del Poder Judicial de la Federación (TEPJF) relacionadas directamente con casos específicos referidos a supuestas afectaciones al ejercicio de la libertad de expresión, considerados paradigmáticos en función de la jurisprudencia que generaron para posteriores reglamentaciones para la actuación de los medios, partidos políticos y candidatos. 
Esta revista forma parte del acervo de la Biblioteca Jurídica Virtual del Instituto de Investigaciones Jurídicas de la UNAM

\section{B. Derecho de réplica}

Consistente en analizar si las resoluciones de la autoridad electoral sobre el ejercicio de este derecho tienen una afectación a la libertad de expresión, ante la ausencia de una ley reglamentaria producto de una omisión legislativa, que provoca una laguna jurídica en los procesos electorales.

\section{Libertad de expresión durante el proceso electoral (actos anticipados de campaña, precampañas y periodo de "intercampañas")}

Que mediante el análisis de casos relevantes y su correspondiente resolución por parte de las autoridades electorales, tuvo como objeto determinar si la norma y las interpretaciones de la autoridad afectan la libertad de expresión y el derecho a la información.

Si bien este llamado nuevo modelo de comunicación política empezó a implementarse en 2009 con las elecciones intermedias, su gran prueba fueron los comicios presidenciales de 2012. En este contexto, la AMEDI consideró importante evaluar la efectividad de este modelo, en virtud de las grandes resistencias que ha tenido por parte de algunos sectores, como también de la expresión de otros grupos que manifiestan la necesidad de contar con elementos concretos para mejorarlo. En este tenor, el estudio y sus líneas de investigación tuvieron como principal objetivo aportar elementos para la discusión pública en aras de asegurar mejores condiciones de equidad en los procesos electorales, y teniendo como base la garantía de los derechos informativos de la sociedad.

Todos los componentes de este proyecto, fueron dirigidos a evaluar la pertinencia del modelo de comunicación político electoral vigente para la democracia mexicana. En este sentido, la sumatoria del análisis de cada una de las líneas de investigación, fue la pauta para elaborar las conclusiones finales.

Diversos organismos internacionales de derechos humanos y libertad de expresión han establecido que los medios de comunicación permiten que los individuos puedan formar su propia opinión. Solamente cuando el individuo es informado podrá evaluar y libremente adherirse a una u otra postura dentro del espectro político. 
Esta revista forma parte del acervo de la Biblioteca Jurídica Virtual del Instituto de Investigaciones Jurídicas de la UNAM

Si bien la ley electoral y el IFE han establecido reglas para procurar que los medios electrónicos cumplan con el derecho a la información y desarrollen una cobertura informativa que favorezca la equidad en las diversas jornadas electorales, lo cierto es que en la actualidad la agenda político electoral también permea a otros programas como los de ficción (telenovelas y series), de entretenimiento (talk shows y matutinos) y a los programas de análisis, debate y opinión, que se han configurado no sólo como espacios para la manifestación de expertos en torno a los temas de la agenda política, sino también como referencia para que los ciudadanos accedan a interpretaciones respecto de propuestas y hechos, y de esta manera, que puedan comprender mejor la información y formarse una opinión respecto de la emisión de su voto, así como escenarios en los cuales las industrias de comunicación fijan su postura frente a actores y partidos políticos.

Respecto de los actores políticos que utilizan los medios para acercarse a la ciudadanía y promover sus campañas, principalmente a través de los spots y en menor medida a través de los debates, podemos decir que son estrategias en las que esencialmente se subsume la propuesta política a la imagen de los candidatos, por lo que la información necesaria para la deliberación pública de las plataformas de gobierno se sustituye por la adjetivación y el escándalo, diluyendo así la cultura democrática.

Aunque se afirma que a raíz de la reforma electoral de 2007-2008 se instauró en nuestro país un nuevo modelo de comunicación políticaelectoral, lo cierto es que hubo un cambio central en la relación de las fuerzas políticas con los medios al excluir la transacción comercial para las campañas y se generaron mejores condiciones de equidad para el acceso a los medios, pero no cambió la manera de comunicarse de los partidos y candidatos con la ciudadanía, como tampoco las estrategias para acercar información de calidad. Finalmente la hegemonía del spot es lo que sigue caracterizando nuestro modelo de comunicación política, en eso el modelo no ha tenido cambios de relevancia.

Por ello concluimos que no es un nuevo modelo de comunicación político electoral, sino que es el mismo modelo que se instauró desde 1997, pero con nuevas reglas. 
Esta revista forma parte del acervo de la Biblioteca Jurídica Virtual del Instituto de Investigaciones Jurídicas de la UNAM

\section{Principales hallazgos de las líneas de investigación}

\section{Observatorio de medios}

\section{A. Contenido y sentido de la propaganda político-electoral durante el proceso electoral de 2012}

El supuesto nuevo modelo de comunicación política apostó por la consolidación del spot como vehículo de la comunicación electoral, con cargo a los tiempos oficiales del Estado.

Con millones de spots en radio y televisión, la ciudadanía estuvo expuesta de manera permanente a las campañas de los partidos y candidatos a la Presidencia de la República, pocos de los cuales fueron para las candidaturas al Congreso o a autoridades locales. Sin embargo, ello no necesariamente se tradujo en mayor información respecto de las propuestas de gobierno y a la deliberación pública de los temas más acuciantes del país.

El material mediático analizado hace posible señalar los siguientes aspectos sobresalientes de las campañas político-electorales:

i. Limitadas en términos de contenido. El formato y la naturaleza intrínseca del spot mediático presentan importantes limitaciones como vehículo de comunicación política. Se trata de una difusión muy acotada de la oferta política, pues se restringe a aquello que pueda ser eficazmente comunicado en pocos segundos.

ii. Viciado en términos discursivos. La exposición de ideas y propuestas políticas se ve opacada ante la fuerza de una retórica argumentativa emocional y una considerable presencia de propaganda de confrontación:

- El modelo de argumentación tiende a apelar en mayor medida a una movilización afectiva asociada a lo inconsciente, más que a propiciar la reflexión ciudadana del voto a partir de información concreta y argumentos racionales. De esta manera, más que contribuir a asegurar el derecho a la información, este modelo tiende a obstaculizarlo.

- Los mensajes de confrontación, cuyo objetivo de comunicación es la desacreditación de los oponentes en la contienda a partir de 
argumentos superficiales de imagen política y percepción social son recurrentes, lo cual deriva en una práctica que llega a naturalizarse como un espacio simbólico de ataques entre candidatos y partidos políticos, lo cual no contribuye necesariamente a la consolidación de una cultura política democrática.

Con todo esto, es posible indicar que el modelo de comunicación política imperante requiere modificaciones que aseguren un proceso electoral que cumpla con la tarea de difundir información concreta, objetiva y racional sobre la oferta electoral como condición para un voto consciente y razonado. Una posibilidad está en promover un modelo no "spotizado", que priorice otras vías de comunicación, como las cápsulas informativas de duraciones razonables entre los dos y tres minutos que permitan plantear propuestas concretas de gobierno.

B. Programas de opinión y análisis y campañas electorales en el marco de la libertad de expresión y derecho a la información

En nuestro país el problema en general del modelo mediático, que rebasa incluso los límites de manejo de la información electoral, es el alto índice de concentración de medios en pocas manos, particularmente en los medios electrónicos, la existencia de programas de análisis y opinión contribuye a la conformación de opiniones diversas respecto a los temas electorales. Sin embargo, cuando la oferta de estos programas proviene especialmente de las dos empresas dominantes en el sector - Televisa y TV Azteca- las opciones de las audiencias se acota a este restringido escenario de fuentes de opinión.

Un aspecto relevante en este mismo sentido es el que observamos en el caso de los llamados medios públicos, debido a su limitada oferta de análisis y opinión respecto al tema electoral en virtud de que pudimos detectar un solo programa de este género, lo cual limita la posibilidad de los ciudadanos para acceder a información y opiniones desde este tipo de medios que pudieran ofertar otro tipo de perspectiva, ya que al no tener fines de lucro pueden contar con mayor libertad y pluralismo. En determinantes para un ejercicio de la democracia como lo son los procesos electorales, la opción que debieran proporcionarnos los medios públicos al abrir programas de opinión y análisis de los asuntos políticos debiera ser una prioridad que genera opciones frente a las ofertas casi únicas de las televisoras privadas comerciales. 
Esta revista forma parte del acervo de la Biblioteca Jurídica Virtual del Instituto de Investigaciones Jurídicas de la UNAM

Cabe resaltar que de los programas analizados, no existe correspondencia entre los tiempos otorgados a los candidatos a la Presidencia de la República ni las tendencias positivas o negativas respectivas con los resultados de la elección, por lo que su influencia directa no puede ser demostrada para ese fin.

Un resultado relevante fue el contraste entre la participación de analistas y conductores en este tipo de programas, ya que tan solo un cercano $20 \%$ corresponde a mujeres, lo cual es un indicador y al mismo tiempo un reflejo de la todavía escasa participación de este sector que representa la mitad del electorado.

Algunas de las propuestas que pueden fortalecer estos ejercicios de libertad de expresión son:

- Incentivar la promoción de programas de análisis y opinión especialmente en los medios públicos que tienen la responsabilidad de contribuir a conformar y estimular la reflexión ciudadana.

- Estos programas contribuyen a generar la reflexión y reconocer que la toma de posiciones y la transparencia de sus tendencias permite y ofrece opciones a los ciudadanos para su propia toma de decisiones.

\section{Los debates presidenciales y el derecho a la información en el proceso electoral de 2012}

En este periodo electoral, el IFE pudo haber determinado globalmente los formatos de ambos debates sobre la base de las recomendaciones que emitió el Comité Técnico de debates, sin embargo, para el primero cedió de manera importante ante los partidos políticos que definieron su formato, quienes cuidando de sobremanera a sus respectivos candidatos y candidata para evitar exponerlos al error, lo limitaron. Esto tuvo como resultado un debate acartonado con una moderación que sólo tuvo un papel menor de apuntar los turnos correspondientes de participación de los contendientes. En este sentido, no fue muy diferente al de elecciones anteriores, donde la principal crítica se centró en que más que debates eran meras exposiciones de los candidatos sin mayor deliberación.

En este sentido, el IFE renunció en buena medida a su mandato como autoridad, pues su papel, más que de negociación con los partidos políticos, es asegurar las mejores condiciones para que la ciuda- 
Esta revista forma parte del acervo de la Biblioteca Jurídica Virtual del Instituto de Investigaciones Jurídicas de la UNAM

danía cuente con elementos para un voto libre, su complacencia en esta situación vulnera la propia institucionalidad y autonomía de un órgano del Estado que debe ser garante de la democracia electoral.

Aunque el formato del segundo debate fue más afortunado, lo cierto es que los candidatos y candidata tuvieron estrategias más enfocadas al ataque y la confrontación que a la explicación de sus propuestas de gobierno. Así, que la ausencia de contenidos se relaciona más con las decisiones de los participantes que con el formato en sí mismo. De este modo, los debates no cumplen con su función a cabalidad porque los candidatos no profundizan en sus propuestas, ganando más su enunciación y los ataques.

Incluso, un punto reiterado que se discute en los programas de pre y pos debate analizados es acerca de quién ganó el encuentro más que las propuestas. La argumentación, como parte de las democracias modernas, queda relegada a quién logra articular mejor los ataques. Entonces, la discusión en los posdebates se trató principalmente de la gestión de la comunicación política por parte de los candidatos y sus equipos y no del debate como un espacio para el fortalecimiento de una cultura política democrática, en donde los políticos están obligados a definir sus planes de gobierno y los ciudadanos a exigir rendición de cuentas.

En este sentido, el modelo actual de comunicación de los partidos, tiene un mayor acento en la mercadotecnia política y mucho menos en la deliberación democrática. Los debates son el momento crucial de la campaña, constituyen el espacio donde los candidatos deberían discutir sus propuestas, sobre todo porque tenemos un esquema basado en los spots que, simplemente por su duración, poco pueden contribuir a ofrecer información para razonar el voto.

$\mathrm{Al}$ igual que en los programas de análisis y opinión, hay una asimetría muy grande en la participación de las mujeres analistas en las mesas posdebate: no llega ni al 15\% el número que estuvieron presentes en estos programas.

En este tenor, se plantean las siguientes propuestas para acercar los debates político-electorales a un modelo de comunicación política donde los ciudadanos obtengan insumos para contar con información de calidad para decidir críticamente su voto:

- Decisión del formato: con el fin de garantizar imparcialidad, el IFE debe asumir su mandato como autoridad en el formato de 
Esta revista forma parte del acervo de la Biblioteca Jurídica Virtual del Instituto de Investigaciones Jurídicas de la UNAM

los debates, con el amparo del marco legal electoral que implique obligatoriedad. En caso de que una candidatura decida no participar en los debates, la sanción puede ser una reducción en porcentaje de los tiempos oficiales para su campaña o bien de su financiamiento público.

- Discusión y puesta en escena: se debe privilegiar un modelo que posibilite la argumentación y la contra-argumentación de propuestas; la réplica debería ser inmediata y no por turnos, pero cuidando la equidad en los tiempos. El formato de panel de expertos o de un moderador activo que pueda pedir a los candidatos que detallen sus propuestas, podría contribuir en este sentido, siempre y cuando no emitan comentarios o juicios sobre los planteamientos de los candidatos.

- Interacción entre los candidatos: la confrontación es parte de la vida política; sin embargo, si se lograra que parte del tiempo oficial empleado para la difusión de spots se utilizara para el desarrollo de mesas de discusión entre los candidatos, especialistas y los equipos de campaña, se podría exhortar a los candidatos para que, en los debates oficiales organizados por el IFE, destinaran el tiempo a la presentación y explicación de su proyecto de gobierno.

- Participación ciudadana: es importante recuperar las propuestas del Comité Técnico del IFE para facilitar la interacción de los candidatos con la ciudadanía. Es necesario buscar alternativas sobre todo con las nuevas tecnologías en redes sociales, para incluir a los ciudadanos en este espacio de comunicación política.

- Cobertura: en cumplimiento al derecho a la información y para garantizar que la mayor parte de la ciudadanía tenga acceso a los debates, debe ser obligatorio que los concesionarios de la televisión privada comercial destinen su canal de mayor cobertura y audiencia a sus transmisiones. El incumplimiento de esta disposición debería tener consecuencias en su título de concesión o bien multas basadas en un porcentaje sobre sus ganancias totales anuales, a fin de evitar hechos tan cuestionables como el de TV Azteca con el primer debate. 


\section{Análisis jurídico: la norma electoral y las resoluciones de las autoridades en materia de libertad de expresión}

La norma jurídica como instrumento de la acción del derecho se diferencia de la norma de presión social, en el hecho de que las sanciones jurídicas son institucionalizadas, mientras que las que siguen a la violación de los usos sociales se confían a la iniciativa incontrolada de los particulares y presentan por ello un grado de aleatoriedad, de inconstancia y de arbitrio mucho mayor que las sanciones jurídicas, las cuales, por estar institucionalizadas, disfrutan de un mayor grado de certeza, no sólo en lo que se refiere a su carácter y entidad, sino a su efectiva aplicación. ${ }^{1}$

Sobre esta base es que se analizó si el actual modelo de comunicación político electoral derivado de la reforma constitucional respeta o violenta el pleno ejercicio de la libertad de expresión y el derecho a la información de los ciudadanos, con la intención de establecer recomendaciones que sirvan a las instancias del Estado mexicano para la mejor aplicación de la norma en aras de fortalecer los procesos democráticos.

Consecuente, la segunda parte de esta investigación tuvo como propósito analizar en el periodo 2009-2012, los límites a la libertad de expresión en la interpretación de las autoridades electorales, el ejercicio del derecho de réplica, los debates y su vinculación con la libertad de expresión y el ejercicio de esta última durante el proceso electoral que incluye a los actos anticipados de campaña, precampañas y periodo conocido como de intercampañas, destacando por su relevancia el ejercicio mediático y la participación de las autoridades en torno a la elección de la Presidencia de la República, así como las principales resoluciones de la autoridad electoral, directamente relacionadas con la libertad de expresión, en casos que se determinaron como paradigmáticos por el alcance de sus resoluciones.

De manera general podemos observar en los casos analizados estrategias recurrentes de los partidos políticos para violar las normas que ellos mismos aprobaron en el Congreso poniendo en serio riesgo

1 Escobar de la Serna, Luis, Principios del derecho de la información, Madrid, Dykinson, 2000 , pp. 23 y 27. 
Esta revista forma parte del acervo de la Biblioteca Jurídica Virtual del Instituto de Investigaciones Jurídicas de la UNAM

su viabilidad y por lo tanto de los procesos electorales y la democracia misma. Bajo el pretexto de la defensa del legítimo derecho a ejercer la libertad de expresión, como si éste fuera un derecho absoluto, se implementan - con cada vez más frecuencia - estrategias sofisticadas para evadir las prohibiciones con la complicidad de actores estatales o no estatales como los medios de comunicación.

La casuística que genera esta dinámica obliga cada vez más a que las autoridades electorales tengan que hacer uso de la interpretación de la norma, pues es casi una tarea imposible que una ley pueda contener todos los supuestos de violaciones que pudieran hacer los regulados.

La implementación de la norma electoral a partir de su reforma en 2007-2008 se ha caracterizado por una extrema judicialización de los procesos comiciales y una permanente tensión entre las autoridades electorales y los regulados. Si bien muchas de las resoluciones de las autoridades no dejan de estar permeadas por criterios políticos, lo cierto es que existe poca institucionalidad por parte de los actores para acatar la ley.

El hecho de que las sanciones en esa reforma se hayan disminuido considerablemente permite que los actores encuentren más conveniente violar la norma a cambio de ganar el poder, de tal manera que el sistema sancionatorio no alcanza a inhibir las conductas de evasión de la ley: los medios saben que los castigos son amonestaciones o multas que no siempre pagan y que de cualquier manera no está en riesgo su concesión, los partidos saben que a pesar de las multas o amonestaciones de todas maneras si ganan llegarán al poder sin mayores consecuencias.

De esta manera se cumple un ciclo de simulación que por más que se cambie la ley para poner nuevos candados, los mismos partidos encuentran la manera de darles la vuelta, haciendo inacabable la demanda de nuevas reglas.

Sin un cambio sustancial en el comportamiento de los partidos políticos en este sentido, simplemente es ilusorio pensar en procesos democráticos plenos. Si bien es cierto que en la competencia política no existen muchas opciones de promocionarse, al estar concentrada la mayor parte de las frecuencias de radiodifusión en unas cuantas manos que imponen sus condiciones, también debe reconocerse que si eso subsiste es porque esos institutos políticos han sido incapaces de cambiar las leyes en esa materia por temor o connivencia con el poder mediático. 
Esta revista forma parte del acervo de la Biblioteca Jurídica Virtual del Instituto de Investigaciones Jurídicas de la UNAM

\section{Los límites a la libertad de expresión en la interpretación de las autoridades electorales}

A partir de la reforma constitucional en materia electoral, uno de los temas sujetos a mayor debate se refiere a los límites de la libertad de expresión, en relación con la prohibición constitucional para los partidos políticos de contratar o adquirir tiempos en cualquier modalidad en radio y televisión, por sí o a través de terceras personas.

La ratio legis de la reforma, en este apartado, se complementa al precisar que tampoco se podrá contratar propaganda electoral en radio y televisión con la finalidad de influir en las preferencias de los electores.

Por ello, el análisis se centra en aquellas resoluciones consideradas relevantes ${ }^{2}$ por los hechos denunciados, así como por los resultados

2 Cfr. Recursos de Apelación, Expedientes: SUP-RAP-510/2011, SUP-RAP-519/2011, SUP-RAP-528/2011 y SUP-RAP-529/2011 acumulados. Sobre adquisición indebida de tiempos en televisión por parte de la coalición "Nayarit, Paz y Trabajo", en virtud de la transmisión de dos "debates" de los precandidatos a Gobernador de Nayarit Martha Elena García Gómez, Guadalupe Acosta Naranjo y Jorge González González. Recursos de Apelación, Expedientes: SUP-RAP-201/2009 y sus acumulados. Sobre promocionales transmitidos en televisión relacionados a la promoción de la revista TVyNovelas en los que se hace mención a las entrevistas hechas a los CC. Raúl Araiza y Maite Perroni, en las cuales expresan las razones por las que apoyarán las propuestas del Partido Verde Ecologista de México.

Recursos de Apelación, Expedientes: SUP-RAP-18/2012, SUP-RAP-20/2012, SUPRAP-21/2012 y SUP-RAP-47/2012 acumulados. Caso de Juan Manuel Márquez Méndez (boxeador) portó y difundió el emblema que identifica al Partido Revolucionario Institucional en su calzoncillo, no obstante que en Michoacán estaba en curso un proceso electoral ordinario, mismo que se encontraba en el periodo de veda previo a la celebración de la jornada electoral.

Tesis jurisprudencial número VII/2010, basada en el Recurso de Apelación número SUP-RAP-175/2009, por derecho de réplica caso del Partido de la Revolución Democrática y Alberto Picasso Barroel, Denuncia presentada por los CC. Eduardo Arguijo Baldenegro y Alberto Picasso Barroel contra la Empresa Editorial El Sol, S.A. de C.V., editora del periódico "El Norte", por hechos que presumiblemente constituyen infracciones al Código Federal de Instituciones y Procedimientos Electorales.

Expediente SCG/PE/PAN/CG/104/2010 y su acumulado CG/PE/PAN/CG/112/2010. Se trata de un spot y un programa de cinco minutos del Partido del Trabajo donde se promocionaba la imagen y el mensaje político de esa organización y su dirigente, Andrés Manuel López Obrador. La autoridad electoral declaró infundada la queja del Partido Acción Nacional porque la difusión ocurría dentro de las prerrogativas de ese partido (tiempos de Estado) y no por contratación de espacios, porque quien se promocionaba no era 
Esta revista forma parte del acervo de la Biblioteca Jurídica Virtual del Instituto de Investigaciones Jurídicas de la UNAM

de los procedimientos administrativos especiales sancionadores (PES) resueltos por la autoridad administrativa - IFE—, que en un par de casos fueron revocados por el TEPJF. Lo anterior denota, en principio, la discrepancia de los criterios que sustentan las resoluciones de ambas autoridades, pues sólo en el caso de la publicidad integrada se observa una misma línea de pensamiento.

Para seleccionar los tres casos que se revisarían, se tomó en consideración que la litis planteada encuadrara en alguna hipótesis vinculada con la adquisición indebida de tiempos en televisión.

En ninguno de los tres casos sujetos a revisión se violentan los principios de la libertad de expresión; por el contrario, conforme al análisis, se fortalece la hipótesis de que la reforma constitucional establece un marco normativo favorable para asegurar el derecho de los partidos políticos al uso de la radio y la televisión, mediante una adecuada

un precandidato o candidato con el debido registro, y porque ocurrió fuera del proceso electoral legal.

Expediente SCG/PE/PRI/CG/155/PEF/71/2011. Sobre actos anticipados de campaña en contra de Andrés Manuel López Obrador. Expedientes SCG/PE/PVEM/CG/047/ PEF/124/2012 y sus acumulados CG/PE/PVEM/CG/048/PEF/125/2012, SCG/PE/ PVEM/CG/049/PEF/126/2012 y SCG/PE/HSGA/CG/052/PEF/129/2012. Caso en contra del actor Héctor Bonilla y el PRD, PT, Movimiento Ciudadano y Morena por actos anticipados de campaña durante el periodo de "intercampaña", consistentes en la transmisión de un spot de radio y televisión en diversas entidades de la República.

Expediente SCG/PE/PAN/CG/086/PEF/163/2012. Sobre enuncia contra Andrés Manuel López Obrador, el PRD, PT y Movimiento Ciudadano, integrantes de la coalición Movimiento Progresista, por actos anticipados de campaña en la explanada del IFE.

Expediente SCG/PE/PRI/CG/152/PEF/68/2011 y su acumulado SCG/PE/PRD/ CG/153/PEF/69/2011. Sobre denuncia en contra de Josefina Vázquez Mota por actos anticipados de campaña. Expediente SCG/PE/PRI/CG/018/PEF/95/2012 en contra de Josefina Vázquez Mota por evento en el Estadio Azul, en pleno periodo de "intercampaña".

Expediente SCG/PE/PRI/CG/061/PEF/138/2012 en contra de la precandidata Josefina Vázquez Mota por acudir al Quinto Informe de Gobierno del estado de Guanajuato.

Expediente SCG/PE/PRD/CG/031/2011 y su acumulado SCG/PE/PAN/CG/051/2011 en contra de Enrique Peña Nieto, por actos anticipados de campaña. Los denunciantes explicaron a la autoridad que de manera sistemática el gobierno del Estado de México, el PRI, sus simpatizantes y el propio Peña Nieto habían estado promoviendo su imagen personal mediante distintas estrategias propagandísticas para lograr el cargo de Presidente de la República.

Expediente SCG/PE/JMEJ/JL/JAL/080/2011 en contra de Enrique Peña Nieto por actos anticipados de campaña por su entrevista concedida al Noticiero de Televisa (XEWTV Canal 2) en la cual el ex gobernador del Estado de México reconoció sus aspiraciones presidenciales de cara a la elección federal de julio de 2012. 
Esta revista forma parte del acervo de la Biblioteca Jurídica Virtual del Instituto de Investigaciones Jurídicas de la UNAM

administración de los tiempos del Estado por parte de la autoridad electoral, en virtud de lo siguiente:

a) Fortalece el margen de actuación de las autoridades electorales, tanto la administrativa como la judicial, al garantizar el libre ejercicio de la libertad de expresión, dentro de los límites que impone el texto constitucional y la regulación secundaria, en lo que corresponde a la prohibición para adquirir indebidamente tiempos en radio y televisión.

b) No hay una sobreinterpretación de la norma constitucional por parte de las autoridades encargadas de resolver las controversias que se suscitan en la contienda electoral (cada una en su esfera de competencia), dado que las diferencias de criterio que sostienen el IFE y el TEPJF son parte de una normalidad interpretativa que deriva de los pesos y contrapesos a que se sujeta el orden constitucional, particularmente en lo que corresponde al principio de división de poderes.

c) El concepto de "sobreinterpretación" es ajeno al lenguaje jurídico, pues el marco constitucional es claro en lo que se refiere a las atribuciones asignadas tanto al IFE como al TEPJF, al grado de señalar que las resoluciones del segundo son inatacables. Lo anterior es entendible bajo la premisa de que los procesos judiciales no pueden prolongarse ad infinitum, de tal suerte que si bien resulta lógico que en un caso específico una de las partes se encuentre insatisfecha con el resultado (aquella a la cual la resolución judicial le es contraria), ello no significa que haya una sobreinterpretación. Estamos en presencia simple y llanamente de la llamada "verdad legal", es decir, aquella verdad que resulta de la valoración, por parte de la autoridad competente, de los elementos contenidos en un expediente judicial, dentro de un proceso que es de estricto derecho.

d) Conforme a lo anterior, resulta meritorio que a partir de la interpretación jurisdiccional se mantenga un criterio único respecto de las hipótesis normativas a que se refiere el antepenúltimo párrafo del artículo 41, Base III, Apartado A, de la Constitución, en lo concerniente a la prohibición de "contratar" o "adquirir" tiempos en radio y televisión.

e) De igual forma, es importante que las autoridades en la materia coincidan en conjugar de manera armónica las hipótesis a que se 
Esta revista forma parte del acervo de la Biblioteca Jurídica Virtual del Instituto de Investigaciones Jurídicas de la UNAM

refiere el punto anterior (contratar o adquirir tiempos en radio y televisión), en cuanto a que, para que sea indebida la contratación de tiempos en radio y televisión, resulta necesario que se trate de propaganda política y que ésta tenga por finalidad influir en el electorado.

También resulta atinado que la interpretación judicial haya permitido, a través de la jurisprudencia, establecer el marco interpretativo que contribuya con las autoridades administrativas (federales y locales) a analizar en mejores condiciones, los atributos a que debe sujetarse la institución jurídica del deslinde para ser efectivo como excluyente de responsabilidad, en aquellos casos en que un instituto político se vea favorecido por actos de terceros en el caso de infracciones normativas que le otorguen un beneficio directo en su imagen o la de sus candidatos.

\section{Derecho de réplica y su vinculación con la libertad de expresión}

Originalmente se pensó que debería ser el IFE quien tendría que ser la autoridad que conociera todos los asuntos relacionados con la réplica en nuestro país, ya que se tenía la falsa idea de que este derecho estaba destinado a los candidatos y partidos políticos, en virtud de la reforma al artículo 6o. constitucional se realizó en el conjunto de reformas en esta materia, sin embargo este derecho va más allá de campañas y candidatos.

El Congreso de la Unión tiene una asignatura pendiente con los mexicanos, se trata de una responsabilidad moral, política y de derecho que aunque no revista una sanción, nos debe llevar a replantear los mecanismos de rendición de cuentas y de responsabilidad legislativa, por lo que se debería insistir en que emitan a la brevedad la ley reglamentaria a que los obliga el artículo 6o., primer párrafo, de la Constitución.

Bajo un enfoque de derechos humanos, ello implicaría necesariamente, incorporar mecanismos de exigibilidad, que contribuyan a reforzar los espacios de fiscalización y control de las políticas legislativas en las estrategias de desarrollo. 
Si bien el IFE no cuenta expresamente con la facultad legal, que si reglamentaria para desahogar los asuntos en esta materia, ha realizado un esfuerzo para garantizar y hacer efectivo el ejercicio del derecho de réplica.

Con el fin de garantizar este derecho se debería observar:

a) Tanto el IFE y el TEPJF deberían elaborar un documento de trabajo para presentar al Congreso, dando cuenta de las dificultades y de las áreas de oportunidad que han encontrado para solventar las quejas relacionadas con el ejercicio de la réplica, para que sirvan de base como un capítulo que se inserte dentro de la norma de carácter legal que emitan para cumplir con la asignatura pendiente.

b) El IFE puede y debe fortalecer el seguimiento de sus asuntos, al ordenar o requerir oficiosamente, sin necesidad de que exista un impulso de parte, información a las partes respecto del cumplimiento de las resoluciones que emita respecto del derecho de réplica, pues se estima que si bien emite una determinación que queda firme, desafortunadamente no cuenta con mecanismos de exigibilidad de los derechos político electorales y mucho menos saber si esas determinaciones se cumplieron.

c) El IFE como el TEPJF, deberían plantear las medidas necesarias para la expeditez del ejercicio de la réplica en materia electoral para que tenga efectos positivos, en caso de ser favorable al quejoso, de lo contrario y aunque sea a su favor la determinación, si pasa demasiado tiempo carece de sentido.

\section{Libertad de expresión durante el proceso electoral: actos anticipados de campaña, precampañas y periodo de intercampañas}

Los actos anticipados de campaña son una figura del derecho electoral mexicano que busca garantizar uno de los principios rectores de la democracia: la equidad en la contienda. Este principio de la democracia mexicana adquiere aún más relevancia por la preeminencia de los medios de comunicación en los procesos electorales y, aún más, por el grado de concentración de la propiedad que exhibe la televisión abier- 
Esta revista forma parte del acervo de la Biblioteca Jurídica Virtual del Instituto de Investigaciones Jurídicas de la UNAM

ta, principal medio de comunicación a través del cual se informan los ciudadanos.

A continuación se enumeran algunos aspectos que resultaron del análisis del marco legal de los actos anticipados de campaña y las resoluciones relevantes de la autoridad en la materia, vinculadas con la libertad de expresión:

a) En particular, los elementos personal, subjetivo y temporal que posibilitan a la autoridad acreditar la existencia de los actos anticipados de campaña sólo tienen un alcance centrado en el proceso electoral legal. Sin embargo, pueden resultar fácilmente sorteables por parte de los actores de la comunicación política debido a que la legislación no puede contemplar un amplio listado de estrategias de comunicación política que escapan del ámbito de acción de la autoridad electoral, como ella misma reconoce.

b) Dos casos de la mayor relevancia para la democracia mexicana, la campaña permanente de Andrés Manuel López Obrador en 2010 y la promoción continua, sistemática y reiterada de Enrique Peña Nieto a través de diversos medios y estrategias de comunicación política mientras fue gobernador del Estado de México, no cancelan la discusión respecto de los alcances y límites de la reforma electoral y lo que pueden hacer o no los partidos y candidatos. En ambos casos, la autoridad resolvió declarar infundados los procedimientos especiales sancionadores, pero permanece en la opinión pública la deliberación de si es lícito o no tener una presencia permanente en los medios de comunicación antes del proceso electoral. Desde luego, el primer criterio debe ser la no utilización de recursos públicos para la promoción personalizada de los funcionarios públicos. Al respecto, resulta urgente reglamentar el artículo 134 constitucional, para que este conjunto de prácticas y conductas de promoción puedan ser reguladas de manera clara y transparente.

c) El derecho a la libertad de expresión no es un derecho absoluto, tiene límites cuando se trata de proteger un interés superior para la sociedad.

d) Llama la atención que el principal instituto político que denunció actos anticipados de campaña fue el Partido Revolucionario Institucional. Sus quejas se formularon en contra tanto de Andrés Manuel López Obrador como de Josefina Vázquez Mota y sus 
respectivos partidos. Por lo tanto, la denuncia se convirtió para el PRI en una estrategia política y jurídica para detener u obstaculizar el avance de sus contrincantes. La denuncia de presuntas irregularidades forma parte de la democracia y de la institucionalidad para resolver diferendos y exigir el complimiento de la ley.

e) Las denuncias por actos anticipados de campaña se configuraron en una estrategia mediática adicional. Cada queja o denuncia (sin que necesariamente los medios y los analistas hicieran el seguimiento puntual de los expedientes y los procedimientos administrativos y jurídicos) trascendieron el ámbito de la autoridad y llegaron a la tribuna de los medios de comunicación, la discusión y la opinión pública, no pocas veces, se litigó y se juzgó en los medios de comunicación, independientemente de los tiempos legales y las decisiones tomadas por los órganos de autoridad responsables de juzgar con base en la ley. Por lo tanto, los medios y los analistas no escaparon a las estrategias de los candidatos, partidos y coaliciones, al mismo tiempo que éstos reaccionaron al veleidoso clima de la opinión pública.

f) En este sentido, el derecho humano fundamental que es la libertad de expresión se convirtió en un paraguas que protegió a todos por igual, sin importar candidato, partido o coalición. Ese es el gran mérito de dicha garantía constitucional y de la reforma electoral.

g) Es claro el cúmulo y crecimiento de quejas y denuncias del proceso electoral federal 2012 con respecto a los anteriores, lo cual satura la capacidad y la eficacia misma del árbitro electoral.

Es de remarcar el papel que han jugado los partidos políticos para el desgaste de la ley y las autoridades electorales, en la revisión de los casos de este estudio, así como en la revisión del contexto general, los institutos políticos tienen una reiterada conducta de violación a las normas que ellos mismos acordaron y aprobaron en el Legislativo, como también fueron omisos para elaborar las leyes reglamentarias para perfeccionar nuestro sistema electoral, violando derechos tan elementales como el de réplica o con su complacencia para que la promoción de gobiernos y funcionarios se siga dando con cargo al erario público.

Si los partidos políticos no son capaces de cumplir sus propias reglas, sería deseable que para inhibir la conducta indebida, se valore el 
Esta revista forma parte del acervo de la Biblioteca Jurídica Virtual del Instituto de Investigaciones Jurídicas de la UNAM

aumento de las sanciones. Sabemos que esta propuesta es controvertida, pero en aras de una supuesta defensa de la libertad de expresión, como si fuera un derecho absoluto, se esconden actos de corrupción y engaño a la autoridad y propiamente a la sociedad.

En conclusión, en los casos revisados, ni la ley electoral ni las autoridades violan la libertad de expresión, pero se requiere de ajustes importantes para que todos los actores la cumplan, como también se requiere cuestionar y deliberar sobre la pobre comunicación de los partidos políticos para con el electorado, y replantear la mejor utilización de los tiempos de Estado.

Pero por más candados que se le pongan al juego democrático, por más funciones que le exijan al IFE para garantizar elecciones libres y equitativas en materia de libertad de expresión y derecho a la información, eso no será posible si no se cambia un problema estructural y originario que es la concentración de medios. El pluralismo de un sistema de medios es el reflejo de su calidad democrática, por ello el de la reforma integral del sistema de medios y su desconcentración sigue siendo el gran pendiente de la transición democrática en México.

\section{Fuentes de consulta}

Expediente SCG/PE/JMEJ/JL/JAL/080/2011 en contra de Enrique Peña Nieto por actos anticipados de campaña por su entrevista concedida al Noticiero de Televisa (XEW-TV Canal 2) en la cual el ex gobernador del Estado de México reconoció sus aspiraciones presidenciales de cara a la elección federal de julio de 2012, disponible en http://www2.ife.org.mx/docs/IFE-v2/DS/DS-CG/ DS-SesionesCG/CG-resoluciones/2011/octubre/CGex201110-27/ CGe271011rp1.2.pdf consultada en marzo de 2013.

Expediente SCG/PE/PAN/CG/086/PEF/163/2012. Sobre enuncia contra Andrés Manuel López Obrador, el PRD, PT y Movimiento Ciudadano, integrantes de la coalición Movimiento Progresista, por actos anticipados de campaña en la explanada del IFE, disponible en http://www2.ife.org. $m x /$ docs/IFE-v2/DS/DS-CG/DS-SesionesCG/ CG-resoluciones/2012/Abril/CGext201204-18/CGe180412rp10-9. $p d f$, consultado en marzo de 2013. 
Esta revista forma parte del acervo de la Biblioteca Jurídica Virtual del Instituto de Investigaciones Jurídicas de la UNAM

Expediente SCG/PE/PAN/CG/104/2010 y su acumulado CG/PE/ PAN/CG/112/2010. Se trata de un spot y un programa de cinco minutos del Partido del Trabajo donde se promocionaba la imagen y el mensaje político de esa organización y su dirigente, Andrés Manuel López Obrador. La autoridad electoral declaró infundada la queja del Partido Acción Nacional porque la difusión ocurría dentro de las prerrogativas de ese partido (tiempos de Estado) y no por contratación de espacios, porque quien se promocionaba no era un precandidato o candidato con el debido registro, y porque ocurrió fuera del proceso electoral legal, disponible en $h t t p: / / w w w . i f e . o r g$. $m x /$ docs/IFE-v2/DS/DS-GacetasElectorales/2011/gaceta-133/pd12. $p d f$ consultado en maro de 2013.

Expediente SCG/PE/PRD/CG/031/2011 y su acumulado SCG/PE/ PAN/CG/051/2011 en contra de Enrique Peña Nieto, por actos anticipados de campaña. Los denunciantes explicaron a la autoridad que de manera sistemática el gobierno del Estado de México, el PRI, sus simpatizantes y el propio Peña Nieto habían estado promoviendo su imagen personal mediante distintas estrategias propagandísticas para lograr el cargo de Presidente de la República, disponible en http://www2.ife.org. $m x /$ docs/IFE-v2/DS/DS-CG/ DS-SesionesCG/CG-resoluciones/2011/Diciembre/CGex201112-14/ CGe141211rp13_1.pdf consultado en marzo de 2013.

Expediente SCG/PE/PRI/CG/018/PEF/95/2012 en contra de Josefina Vázquez Mota por evento en el Estadio Azul, en pleno periodo de "intercampaña", disponible en http://www.ife.org. $m x /$ docs/IFE- $v 2 /$ DS/DS-CG/DS-SesionesCG/CG-actas/2012/Febrero/CGex20120208/CGex201202-08_01.pdf, pág. 1241, consultado en marzo 2013. Expediente SCG/PE/PRI/CG/061/PEF/138/2012 en contra de la precandidata Josefina Vázquez Mota por acudir al Quinto Informe de Gobierno del estado de Guanajuato, disponible en $h t t p: / / w w w . i f e$. org. $m x /$ docs/IFE-v2/DS/DS-CG/DS-SesionesCG/CG-actas/2012/Junio/CGex201206-14/CGex201206-14_01.pdf, la página 223, consultada en marzo de 2013.

Expediente SCG/PE/PRI/CG/152/PEF/68/2011 y su acumulado SCG/ $\mathrm{PE} / \mathrm{PRD} / \mathrm{CG} / 153 / \mathrm{PEF} / 69 / 2011$. Sobre denuncia en contra de Josefina Vázquez Mota por actos anticipados de campaña, disponible en $h t t p: / / w w w . i f e . o r g . m x / d o c s / I F E-v 2 / D S / D S-C G / D S-S e s i o n e s C G /$ CG-actas/2012/Enero/CGex201201-18/CGex201201-18_01.pdf, página 1054, consultada en marzo de 2013. 
Esta revista forma parte del acervo de la Biblioteca Jurídica Virtual del Instituto de Investigaciones Jurídicas de la UNAM

Expediente SCG/PE/PRI/CG/155/PEF/71/2011. Sobre actos anticipados de campaña en contra de Andrés Manuel López Obrador, disponible en http://www.ife.org.mx/docs/IFE-v2/DS/DS-CG/DSSesionesCG/CG-actas/2012/Abril/CGex201204-11/CGex20120411_01.pdf, en la página 560, consultada en marzo de 2013.

Expedientes SCG/PE/PVEM/CG/047/PEF/124/2012 y sus acumulados CG/PE/PVEM/CG/048/PEF/125/2012, SCG/PE/PVEM/CG/049/P $\mathrm{EF} / 126 / 2012$ y SCG/PE/HSGA/CG/052/PEF/129/2012. Caso en contra del actor Héctor Bonilla y el PRD, PT, Movimiento Ciudadano y Morena por actos anticipados de campaña durante el periodo de "intercampaña", consistentes en la transmisión de un spot de radio y televisión en diversas entidades de la República, disponible en $h t t p: / / w w w . i f e . o r g . m x / d o c s / I F E-v 2 / D S / D S-C G / D S$-SesionesCG/ CG-actas/2012/Julio/CGex201207-12/CGex201207-12_01.pdf, en la página 1567, consultada en marzo de 2013.

Recursos de Apelación, Expedientes: SUP-RAP-18/2012, SUPRAP-20/2012, SUP-RAP-21/2012 y SUP-RAP-47/2012 acumulados. Caso de Juan Manuel Márquez Méndez (boxeador) portó y difundió el emblema que identifica al Partido Revolucionario Institucional en su calzoncillo, no obstante que en Michoacán estaba en curso un proceso electoral ordinario, mismo que se encontraba en el periodo de veda previo a la celebración de la jornada electoral, disponible en https://colaboracion.ife.org.mx/Direccion_Juridica/Documentos\%20de\%20Sentencia/APELACIONES/2012/ FEBRERO/SUP-RAP-18-2012\%20PRI\%20Y\%20OTROS\%20NOTIFiCADA\%20EL\%2014\%20DE\%20FEBRERO\%20A\%20LAS\%20 12\%20HORAS\%20CON\%2021\%20MINUTOS.pdf , consultada en marzo de 2013.

Recursos de Apelación, Expedientes: SUP-RAP-201/2009 y sus acumulados. Sobre promocionales transmitidos en televisión relacionados a la promoción de la revista TVyNovelas en los que se hace mención a las entrevistas hechas a los CC. Raúl Araiza y Maite Perroni, en las cuales expresan las razones por las que apoyarán las propuestas del Partido Verde Ecologista de México, disponible en: http://www.ife.org.mx/docs/IFE-v2/DS/DS-CG/DS-SesionesCG/CGacuerdos/2009/agosto/11agosto/CGe110809rp8.pdf, consultado en marzo de 2013.

Recursos de Apelación, Expedientes: SUP-RAP-510/2011, SUPRAP-519/2011, SUP-RAP-528/2011 y SUP-RAP-529/2011 acu- 
Esta revista forma parte del acervo de la Biblioteca Jurídica Virtual del Instituto de Investigaciones Jurídicas de la UNAM

mulados. Sobre adquisición indebida de tiempos en televisión por parte de la coalición "Nayarit, Paz y Trabajo", en virtud de la transmisión de dos "debates" de los precandidatos a Gobernador de Nayarit Martha Elena García Gómez, Guadalupe Acosta Naranjo y Jorge González González, disponible en https://colaboracion.ife. org.mx/Direccion_Juridica/Documentos\%20de\%20Sentencia/APELACIONES/2011/NOVIEMBRE/SUP-RAP-510-2011\%20PAN\%20 Y\%20OTROS\%20NOTIF iCADA\%20EL\%2030\%20DE\%20NOVIEMBRE\%20A\%20LAS\%2019\%20HORAS\%20CON\%2050\%20 MINUTOS\%202\%20DE\%202.pdf, consultada en marzo de 2013 Tesis jurisprudencial número VII/2010, basada en el Recurso de Apelación número SUP-RAP-175/2009, por derecho de réplica caso del Partido de la Revolución Democrática y Alberto Picasso Barroel, Denuncia presentada por los CC. Eduardo Arguijo Baldenegro y Alberto Picasso Barroel contra la Empresa Editorial El Sol, S.A. de C.V., editora del periódico "El Norte", por hechos que presumiblemente constituyen infracciones al Código Federal de Instituciones y Procedimientos Electorales, disponible en $h t t p: / / w w w . o r d e n j u r i-$ dico.gob.mx/Publicaciones/CDs2010/CDLGSMIME/pdf/A86-1,\%20 d),\%20T-1.pdf, consultada en marzo de 2013. 